\title{
FACTORS ASSOCIATED WITH PARENTAL REFUSAL OF ROUTINE VACCINATION IN THE CZECH REPUBLIC
}

\author{
Jana Dáňová, Jiří Šálek, Aneta Kocourková, Alexander M. Čelko \\ Third Faculty of Medicine, Charles University, Prague, Czech Republic
}

\section{SUMMARY}

Aim: Routine vaccination is one of the most important preventive methods which is responsible for the decreasing trend of morbidity and mortality of vaccine preventable infectious diseases, their complications and sequelae. The impact of vaccination on declining trend of these diseases is well known and confirmed by a large number of epidemiological studies. In the Czech Republic, there is high vaccination coverage in regards to most vaccine preventable diseases. However, during the last decade proportion of parents refusing routine vaccination of their children due to different factors is increasing. The presented study evaluates current situation in the Czech Republic and describes the most significant factors in parents decision making.

Methods: The study was conducted between 1 July 2013 and 31 March 2014 as a questionnaire based survey (cross-sectional study). The questionnaire was created with multiple choice answers. Questions were addressed to parents or legal representatives of children aged 0-18 years. Types of questions were divided into several subgroups. The study was performed in the Czech Republic in two different districts of Prague and Zlín.

Results: In the sample size $(n=480)$ we detected 11 parents who refused vaccination of 11 children $(2.29 \%)$. The most often refused vaccines in the prevalence study were hexavaccine (1st dose) and measles, mumps and rubella vaccine (1st dose). The hexavaccine includes tetanic anatoxin, diphtheric anatoxin, acellular pertussis vaccine, conjugate vaccine against Haemophilus influenzae $b$, inactivated polio vaccine, and recombinant vaccine against viral hepatitis $B$. The measles, mumps, rubella vaccine contains live attenuated viruses of measles, mumps, rubella.

Conclusion: We observed increasing trend of routine vaccination refusal in children during the last ten years (compared to situation in the year $2004 p<0.001)$. The most important factors associated with this progression were distrust to vaccination, fear of some vaccine components and fear of adverse reactions.

Key words: routine vaccination, refusal of vaccination, vaccine preventable disease, parents, children

Address for correspondence: J. Dáňová, Dept. of Epidemiology, Third Faculty of Medicine, Charles University, Ruská 87, 10000 Prague 10, Czech Republic. E-mail: jana.danova@|f3.cuni.cz

\section{INTRODUCTION}

Vaccination represents one of the most effective public health interventions in developed and developing countries. Furthermore, vaccination prevents large number of serious illnesses and deaths from vaccine preventable diseases all over the world. However, even in high income countries an indispensable proportion of children remains unvaccinated against some of vaccine preventable diseases due to a number of reasons (1). First, the health system may fail to offer the vaccination of children and/or fail to invite them, or parents may forget to take their children for vaccination and thus fail to complete the full schedule. Second, the children may not be vaccinated because they have genuine or suspected contraindications. Third, which seems to be significant nowadays in the Czech Republic, parents may refuse vaccination due to factors concerning the safety of some vaccines. The Czech Republic is a country with traditionally well-organized routine vaccination and reports high vaccination coverage. It is likely that results from the Czech Republic should indicate rates of vaccine refusal among parents in the country with well implemented immunisation programme as well as detect the most important factors leading to these findings (2). Vaccination coverage represents most important factor in herd protection of population against vaccine preventable diseases. In the study we evaluated the proportion of parents refusing vaccination of their children. The main objective of the study was to describe characteristics which are associated with common attitude of parents to vaccination. In our study we have tried to answer the following questions: Is it true that the attitude of parents to the strategy of routine vaccination in the Czech Republic has changed in the last few years? What is the frequency of refusal of vaccination from the parents? What are the reasons for refusal?

\section{MATERIALS AND METHODS}

The study was conducted in two districts of the Czech Republic, Prague (the capital city) and Zlín (a town with a population of 76,000) between 1 July 2013 and 31 March 2014. We created structured questionnaire using multiple choice answers and questions were divided into several subgroups: personal data of parents or legal representatives - age, gender, educational level, socioeconomic status; sources of information about vaccination strategy for parents; reasons for refusal of any type of vaccination in children. The most important part of questions focused 
on attitudes of parents or legal representatives to organisation of vaccination and legislative regulations in the Czech Republic. Main emphasis was placed on factors of vaccination refusal due to different reasons. The study procedures were approved by the ethical committee of the Third Faculty of Medicine, Charles University. Questionnaires were addressed to parents of children in the age group from 0-18 years and were distributed in paediatricians' offices, kindergartens, schools, and leisure time clubs which were randomly selected in both regions. The distribution of questionnaires was under the supervision of project team members. From collected information we estimated the following indices: the number of parents who refused vaccination of their children, their proportion of whole study group, proportions of specific type of vaccines which were refused. We also examined reasons associated with refusal of vaccinations from parents as distrust to vaccination, specific lifestyle, fear of adverse reactions, religious reasons, adverse reaction after previous vaccination, fear of components in a vaccine, or low risk of infection. We also asked parents where they had been looking for information about vaccinations (internet, media, recommendations of persons not medically educated, recommendations of professionals, scientific publications). Data from questionnaires were computed and analysed in PC program Stata and Microsoft Excel. Mainly descriptive statistics were used. The differences in distribution of vaccination refusal in two study groups were tested by Yates chi $^{2}$ test.

\section{RESULTS}

Within the study we distributed 750 questionnaires and received 480 filled in questionnaires back. The response rate was $64 \%$. The age of parents participating in the study differed from 19-49 years among females (mean age 34.7) and from 20-63 years among males (mean age 37.4). Specific age of parents was not statistically significant in refusal of children vaccination. In the sample size we detected 11 parents who refused vaccination of their 11 children $(2.29 \%)$ (Table 1$)$. The mostly refused vaccines in this cross-sectional study were the 1st dose of hexavaccine (7x) and the 1st dose of measles, mumps, rubella vaccine (7x). We compared our data with the study carried out in 2004 (3). We found statistical significance in increasing trend of vaccination refusal in the Czech Republic (Table 2). Main reasons associated with refusal of vaccination were fear of components contained in the vaccine and fear of adverse reactions (Table 3). A significant difference was found in sources of information obtained by parents who wanted to get their children vaccinated and parents who refused it. Parents who refused to get the child vaccinated mostly gathered information from the Internet (55\%), while parents preferring vaccination mostly looked for information from the specialist (58\%). We did not find any statistically significant difference between data collected in Prague and Zlín.

Table 1. Frequency of routine vaccination refusal

\begin{tabular}{|l|c|c|}
\hline Refusal of routine vaccination & Number & $\%$ \\
\hline Yes & 11 & 2.29 \\
\hline No & 469 & 97.71 \\
\hline Total & 480 & 100.00 \\
\hline
\end{tabular}

Table 2. Comparison of two studies focused on refusal of vaccination in the Czech Republic

\begin{tabular}{|l|c|c|}
\hline & Study 2004 & Study 2014 \\
\hline Number of vaccinated children & 5,036 & 469 \\
\hline Number of refused vaccination & 2 & 11 \\
\hline Total & 5,038 & 480 \\
\hline
\end{tabular}

Yates chi test $=85.22, p<0.001$

Table 3. Reasons for vaccination refusal

\begin{tabular}{|l|c|c|}
\hline & Number & $\%$ \\
\hline Distrust of vaccination & 5 & 15.15 \\
\hline Specific life style & 6 & 18.18 \\
\hline Fear of adverse reactions & 7 & 21.21 \\
\hline Religious factors & 1 & 3.03 \\
\hline Adverse reaction after previous vaccination & 1 & 3.03 \\
\hline Fear of components in the vaccine & 7 & 21.21 \\
\hline Low probability of infection & 6 & 18.18 \\
\hline Total & 33 & 100.00 \\
\hline
\end{tabular}

More than one reason for vaccination refusal was detected in 11 cases

\section{DISCUSSION}

The study attempted to estimate population rates of routine vaccination refusal in children. We observed $2.29 \%$ cases of refusal, which is statistically significant increase in trend over the last 10 years (3). Studies conducted in many developed countries also detected increasing trend in refusal of routine vaccination of children. In 2010, McCauley et al. conducted a telephone survey among 1,500 parents of 6-23 months old children which confirmed $3.4 \%$ of children without any vaccination. The most common reason was "fear of serious side effects" (4). Parents of children who refuse routine vaccination are usually obtaining information about vaccination hazards from websites. Unfortunately, many of these websites are created by groups of people refusing vaccination in principle. What is important to do if parents refuse immunization describes an article by Diekema (5). It is essential to explain advantages of vaccination strategy, the paediatrician should share honestly what is and is not known about the risks and benefits of the vaccine and correct any misinformation (5). Refusal of vaccination can result in inadequate vaccine coverage (6). Authors of the French recently published study emphasize the importance of sufficient level of vaccination coverage (95\%). A decrease of this level could be associated with outbreaks of vaccine preventable diseases as was for example the recent measles outbreak in France and other European countries (7). The outbreak of measles in our country (spring 2014) should be sufficient warning. According to the study finished in the United States in 2013 it is clear that the level of vaccination coverage is associated with demographic characteristics. Children living at or below the poverty line have lower vaccination uptake and are at higher risk of various preventable diseases (8). For example, the risks of encephalopathy related to the measles vaccine is 1 in 1 million, while the risk of encephalopathy in case of measles is 1,000 times greater (9). Outbreaks of vaccine-preventable disease often start among persons who refused vaccination. The US 
studies have indicated that children who are under-vaccinated are likely to belong to families that intentionally refuse vaccines (10). In the United States there were five measles outbreaks reported in 2008. All persons with measles were either unvaccinated or did not have evidence of immunization (8). This should be an important warning for our country. It is evident that high level of herd protection due to vaccination coverage is the essential factor in protection against vaccine preventable diseases (6). In our study we found association between sources of information and refusal of vaccination. Refusing parents have been mostly looking for information about vaccination on web pages. Unfortunately there are many false statements, one specific adverse reaction is presented as common effect of vaccination. What is the crucial factor to stop increasing trend of vaccination refusal? We supposed that systematic education of target population can help to understand the importance of immunization and help to form large enough herd protection. Limitation of the study is probably the lack of more detailed data, especially socioeconomic data of parents responding to questionnaires. These data would allow the validation of specific parameters of vaccination refusal. Representativeness of the study should be also considered. The study was conducted in two districts of the Czech Republic (Zlín, Prague). Since the participating population was not nationally representative, we try to compare two different types of regions of the Czech Republic. Prague is the capital city with 1.27 million residents and Zlín is a town with a population of 76,000 in the Moravia region. Anyway, we believe that our results could predict the real trend of refusal of routine vaccination in our country. For further strategy of immunization it is important to know that vaccines are very safe, but they are not risk free and they are not $100 \%$ effective (11, 12). The inevitable factor for vaccination strategy is cooperation of health professionals with parents of children.

\section{CONCLUSION}

The literature published in different countries on the frequency of vaccination refusal showed similar trend as the one observed in the Czech Republic. Refusal of routine vaccination observed in our study was $2.29 \%$, a significant increase over the last 10 years $(p=0.001)$. Important are sources of information about vaccination in various groups of parents. Parents who agree with vaccination mostly rely on specialists' recommendation. Parents refusing vaccination prefer the Internet as a source of information. Mainstream media and the Internet promoted by celebrity opinion often give a greater weight to ill-informed opinion compared with scientific studies proving safety and effectiveness of the vaccines. And this misinformation leads to parental concern. The crucial role of healthcare providers is to understand these concerns of parents in order to effectively deal with them and help parents in choosing the most proper protection of their children against vaccine preventable infectious diseases.

\section{Acknowledgements}

The study was developed as a part of the project PRVOUK (P 02). The Charles University Research Development Schemes (known under the Czech acronym PRVOUK) have been providing institutional support for research at Charles University since 2012.

\section{Conflict of Interests}

None declared

\section{REFERENCES}

1. Bedford H, Elliman D. Concerns about immunisation. BMJ. 2000 Jan 22;320(7229):240-3.

2. Kříž B, Beneš Č, Částková J, Šrámová H, Švandová E. Serological survey of the antibodies against selected infectious diseases in the Czech Republic, 2001 year. Cent Eur J Public Health. 2003 Dec;11 Suppl:1-67.

3. Danova J, Gopfertova D, Bobak M. Rates of contraindications and use of alternative vaccines in routine immunisation o children: a population based study in the Czech Republic. Vaccine. 2007 May 10;25(19):3890-5.

4. McCauley MM, Kennedy A, Basket M, Sheedy K. Exploring the choice to refuse or delay vaccines: a national survey of parents of 6-through 23-month-olds. Acad Pediatr. 2012 Sep-Oct;12(5):375-83.

5. Diekema DS; American Academy of Pediatrics Committee on Bioethics. Responding to parental refusals of immunization of children. Pediatrics. 2005 May; 115(5):1428-31.

6. Harmsen IA, Mollema L, Ruiter RA, Paulussen TG, de Melker HE, Kok G. Why parents refuse childhood vaccination: a qualitative study using online focus groups. BMC Public Health. 2013 Dec 16;13:1183. doi: 10.1186/1471-2458-13-1183.

7. Bégué P. Consequences of opposition to vaccination in France and Europe. How to maintain effective vaccine coverage in 2010? Bull Acad Natl Med. 2010 Apr-May;194(4-5):719-32. (In French.)

8. Leib S, Liberatos P, Edwards K. Pediatricians' experience with and response to parental vaccine safety concerns and vaccine refusals: a survey of Connecticut pediatricians. Public Health Rep. 2011 Jul-Aug;126 Suppl 2:13-23.

9. Elam-Evans LD, Yankey D, Singleton JA, Kolasa M; Centers for Disease Control and Prevention (CDC). National, state, and selected local area vaccination coverage among children aged 19-35 months - United States, 2013. MMWR Morb Mortal Wkly Rep. 2014 Aug 29;63(34):741-8.

10. Jacobson RM. Vaccination refusal and parental education: lessons learnt and future challenges. Pediatr Health. 2010;4(3):239-42.

11. Omer SB, Salmon DA, Orenstein WA, deHart MP, Halsey N. Vaccine refusal, mandatory immunization and the risks of vaccine-prevenable diseases. N Engl J Med. 2009 May 7;360(19):1981-8.

12. Grossman Z, van Esso D, Del Torso S, Hadjipanayis A, Drabik A, Gerber A, et al. Primary care pediatricians' perceptions of vaccine refusal in Europe. Pediatr Infect Dis J. 2011 Mar;30(3):255-6. 